\title{
Fuzzy Geometric Programming in Multivariate Stratified Sample Surveys in Presence of Non-Response with Quadratic Cost Function
}

\author{
Shafiullah' ${ }^{1}$, Mohammad Faisal Khan' ${ }^{2}$, Irfan Ali ${ }^{1}$ \\ ${ }^{1}$ Department of Statistics \& Operations Research, Aligarh Muslim University, Aligarh, India \\ ${ }^{2}$ Department of Computing and Informatics Saudi Electronics University, Riyadh, Saudi Arabia \\ Email: shafi.stats@gmail.com, faisalkhan004@yahoo.com, irfii.ali@gmail.com
}

Received 19 March 2014; revised 19 April 2014; accepted 26 April 2014

Copyright (C) 2014 by authors and Scientific Research Publishing Inc.

This work is licensed under the Creative Commons Attribution International License (CC BY). http://creativecommons.org/licenses/by/4.0/

\section{(c) (i) Dpen Access}

\begin{abstract}
In this paper, the problem of non-response with significant travel costs in multivariate stratified sample surveys has been formulated of as a Multi-Objective Geometric Programming Problem (MOGPP). The fuzzy programming approach has been described for solving the formulated MOGPP. The formulated MOGPP has been solved with the help of LINGO Software and the dual solution is obtained. The optimum allocations of sample sizes of respondents and non respondents are obtained with the help of dual solutions and primal-dual relationship theorem. A numerical example is given to illustrate the procedure.
\end{abstract}

\section{Keywords}

Geometric Programming, Fuzzy Programming, Non-Response with Travel Cost, Optimum Allocations, Multivariate Stratified Sample Surveys

\section{Introduction}

In sampling the precision of an estimator of the population parameters depends on the size of the sample and variability among the units of the population. If the population is heterogeneous and size of the sample depends on the cost of the survey, then it is likely to be impossible to get a sufficiently precise estimate with the help of simple random sampling from the entire population. In order to estimate the population mean or total with greater precision, the heterogeneous population is divided into mutually-exclusive, exhaustive and non-overlap- 
ping strata which will be more homogeneous than the entire population. The entire population is called Stratified Random Sampling. The problem of optimum allocation in stratified random sampling for univariate population is well known in sampling literature; see for example Cochran [1] and Sukhatme et al. [2]. In multivariate stratified sample surveys problems the non-response can appear when the required data are not obtained. The problem of non-response may occur due to the refusal by respondents or their not being at home, making the information of sample inaccessible. The problem of non-response occurs in almost all surveys. The extent of nonresponse depends on various factors such as type of the target population, type of the survey and the time of survey. For the problem of non-response in stratified sampling it may be assumed that every stratum is divided into two mutually exclusive and exhaustive groups of respondents and non respondents.

Hansen and Hurwitz [3] presented a classical non-response theory which was first developed for surveys in which the first attempt was made by mailing the questionnaires and a second attempt was made by personal interviews to a sub sample of the non respondents. They constructed the estimator for the population mean and derived the expression for its variance and also worked out the optimum sampling fraction among the non respondents. El-Badry [4] further extended the Hansen and Hurwitz's technique by sending waves of questionnaires to the non respondent units to increase the response rate. The generalized El-Badry's approach for different sampling design was given by Foradori [5]. Srinath [6] suggested the selection of sub samples by making several attempts. Khare [7] investigated the problem of optimum allocation in stratified sampling in presence of nonresponse for fixed cost as well as for fixed precision of the estimate. Khan et al. [8] suggested a technique for the problem of determining the optimum allocation and the optimum sizes of subsamples to various strata in multivariate stratified sampling in presence of non-response which is formulated as a Nonlinear Programming Problem (NLPP). Varshney et al. [9] formulated the multivariate stratified random sampling in the presence of non-response as a Multi-objective Integer Nonlinear Programming problem and a solution procedure is developed using lexicographic goal programming technique to determine the compromise allocation. Fatima and Ahsan [10] addressed the problem of optimum allocation in stratified sampling in the presence of non-response and formulated as an All Integer Nonlinear Programming Problem (AINLPP). Varshney et al. [11] have considered the multivariate stratified population with unknown strata weights and an optimum sampling design is proposed in the presence of non-response to estimate the unknown population means using DSS strategy and developed a solution procedure using Goal Programming technique and obtained an integer solution directly by the optimization software LINGO. Raghav et al. [12] has discussed the various multi-objective optimization techniques in the multivariate stratified sample surveys in case of non-response.

Geometric Programming (GP) is a smooth, systematic and an effective non-linear programming method used for solving the problems of sample surveys, management, transportation, engineering design etc. that takes the form of convex programming. The convex programming problems occurring in GP are generally represented by an exponential or power function. GP has certain advantages over the other optimization methods because it is usually much simpler to work with the dual than the primal one. The degree of difficulty (DD) plays a significant role for solving a non-linear programming problem by GP method.

Geometric Programming (GP) has been known as an optimization tool for solving the problems in various fields from 1960's. Duffin, Peterson and Zener [13] and also Zener [14] have discussed the basic concepts and theories of GP with application in engineering in their books. Beightler, C.S., and Phililps, D.T. [15], have also published a famous book on GP and its application. Davis and Rudolph [16] applied GP to optimal allocation of integrated samples in quality control. Ahmed and Charles [17] applied geometric programming to obtain the optimum allocation problems in multivariate double sampling. Ojha, A.K. and Das, A.K. [18] have taken the MultiObjective Geometric Programming Problem being cost coefficient as continuous function with weighted mean and used the geometric programming technique for the solutions. Maqbool et al. [19] and Shafiullah et al. [20] have discussed the geometric programming approach to find the optimum allocations in multivariate two-stage sampling and three-stage sample surveys respectively.

In many real-world decision-making problems of sample surveys, environmental, social, economical and technical areas are of multiple-objectives. It is significant to realize that multiple objectives are often non-commensurable and in conflict with each other in optimization problems. The multi-objective models with fuzzy objectives are more realistic than deterministic of it. The concept of fuzzy set theory was firstly given by Zadeh [21]. Later on, Bellman and Zadeh [22] used the fuzzy set theory for the decision-making problem. Tanaka et al. [23] introduces the objective as fuzzy goal over the $\alpha$-cut of a fuzzy constraint set and Zimmermann [24] gave the concept to solve multi-objective linear-programming problem. Biswal [25] and Verma [26] developed fuzzy 
geometric programming technique to solve Multi-Objective Geometric Programming (MOGP) problem. Islam [27] [28] has discussed modified geometric programming problem and its applications and also another fuzzy geometric programming technique to solve MOGPP and its applications. Fuzzy mathematical programming has been applied to several fields.

In this paper, we have formulated the problem of non-response with significant travel cost where the cost is quadratic in $\sqrt{n_{h}}$ in multivariate stratified sample surveys as a Multi-Objective Geometric Programming problem (MOGPP). The fuzzy programming approach has been described for solving the formulated MOGPP and the optimum allocations of sample sizes of respondents and non respondents are obtained. A numerical example is given to illustrate the procedure.

\section{Formulation of the Problem}

In stratified sampling the population of $N$ units is first divided into $L$ non-overlapping subpopulation called strata, of sizes $N_{1}, N_{2}, \cdots, N_{h}, \cdots, N_{L}$ with $\sum_{h=1}^{L} N_{h}=N$ and the respective sample sizes within strata are drawn with independent simple random sampling denoted by $n_{1}, n_{2}, \cdots, n_{h}, \cdots, n_{L}$ with $\sum_{h=1}^{L} n_{h}=n$.

Let for the $h^{\text {th }}$ stratum:

$N_{h}$ : Stratum size.

$\bar{Y}_{h}:$ Stratum mean.

$S_{h}^{2}$ : Stratum variance.

$\hat{W}_{h 1}=\hat{N}_{h 1} / N$ : the estimated stratum weight among respondents.

$\hat{W}_{h 2}=\hat{N}_{h 2} / N$ : the estimated stratum weight among non-respondents.

$N_{h 1}$ : the sizes of the respondents.

$N_{h 2}=N_{h}-N_{h 1}$ : the sizes of non respondents groups.

$n_{h}$ : Units are drawn from the $h^{\text {th }}$ stratum. Further let out of $n_{h}, n_{h 1}$ units belong to the respondents group.

$n_{h 2}=n_{h}-n_{h 1}$ : Units belong to the non respondents group.

$n=\sum_{h=1}^{L} n_{h}$ : The total sample size.

A more careful second attempt is made to obtain information on a random subsample of size $r_{h}$ out of $n_{h 2}$ non respondents for the representation from the non respondents group of the sample.

$r_{h}=n_{h 2} / k_{h} ; h=1,2, \cdots, L$ : Subsamples of sizes at the second attempt to be drawn from $n_{h 2}$ non-respondent group of the $h^{\text {th }}$ stratum. Where $k_{h} \geq 1$ and $1 / k_{h}$ denote the sampling fraction among non respondents. Since $N_{h 1}$ and $N_{h 2}$ are random variables hence their unbiased estimates are given as:

$\hat{N}_{h 1}=n_{h 1} N_{h} / n_{h}$ : The unbiased estimates of the respondents group.

$\hat{N}_{h 2}=n_{h 2} N_{h} / n_{h}$ : The unbiased estimate of the non respondents group.

$\bar{y}_{j h 1} ; j=1, \cdots, p$ : denotes the sample means of $j^{\text {th }}$ characteristic measured on the $n_{h 1}$ respondents at the first attempt.

$\bar{y}_{\text {jh2 }\left(r_{h}\right.} ; j=1, \cdots, p$ : denotes the $r_{h}$ sub sampled units from non respondents at the second attempt.

Using the estimator of Hansen and Hurwitz [3], the stratum mean $\bar{Y}_{j h}$ for $j^{\text {th }}$ characteristic in the $h^{\text {th }}$ stratum may be estimated by

$$
\bar{y}_{j h(w)}=\frac{n_{h 1} \bar{y}_{j h 1}+n_{h 2} \bar{y}_{j h 2\left(r_{h}\right)}}{n_{h}}
$$

It can be seen that $\bar{y}_{j h(w)}$ is an unbiased estimate of the stratum mean $\bar{Y}_{j h}$ of the $h^{\text {th }}$ stratum for the $j^{\text {th }}$ characteristic with a variance.

$$
v\left(\bar{y}_{j h(w)}\right)=\left(\frac{1}{n_{h}}-\frac{1}{N_{h}}\right) S_{j h}^{2}+\frac{W_{h 2}^{2} S_{j h 2}^{2}}{r_{h}}-\frac{W_{h 2}^{2} S_{j h 2}^{2}}{n_{h}}
$$

where $S_{j h}^{2}$ is the stratum variance of $j^{\text {th }}$ characteristic in the $h^{\text {th }}$ stratum; $j=1,2, \cdots, p$ and $h=1,2, \cdots, L$ given as:

$$
S_{j h}^{2}=\frac{1}{N_{h}-1} \sum_{i=1}^{N_{h}}\left(y_{j h i}-\bar{Y}_{j h}\right)^{2}
$$


where $y_{j h i}$ denote the value of the $i^{\text {th }}$ unit of the $h^{\text {th }}$ stratum for $j^{\text {th }}$ characteristic.

$\bar{Y}_{j h}=1 / N_{h} \sum_{i=1}^{N_{h}} y_{j h i}$ : is the stratum mean of $y_{j h i}$.

$S_{j h 2}^{2}$ is the stratum variance of the $j^{\text {th }}$ characteristic in the $h^{\text {th }}$ stratum among non respondents, given by:

$$
\hat{S}_{j h 2}^{2}=\frac{1}{\hat{N}_{h 2}-1} \sum_{i=1}^{\hat{N}_{h 2}}\left(y_{j h i}-\bar{Y}_{j h 2}\right)^{2}
$$

$\bar{Y}_{j h 2}=1 / \hat{N}_{h 2} \sum_{i=1}^{\hat{N}_{h 2}} y_{j h i}$ is the stratum mean of $y_{j h i}$ among non respondents.

$\hat{W}_{h 2}=\hat{N}_{h 2} / N_{h}$ is stratum weight of non respondents in $h^{\text {th }}$ stratum.

If the true values of $S_{j h}^{2}$ and $S_{j h 2}^{2}$ are not known they can be estimated through a preliminary sample or the value of some previous occasion, if available, may be used.

Furthermore, the variance of $\bar{y}_{j(w)}=\sum_{h=1}^{L} W_{h} \bar{y}_{j h(w)}$, (ignoring fpc) is given as:

$$
V\left(\bar{y}_{j(w)}\right)=\sum_{h=1}^{L} W_{h}^{2} v\left(\bar{y}_{j h(w)}\right)=\sum_{h=1}^{L} \frac{W_{h}^{2}\left(S_{j h}^{2}-\hat{W}_{h 2} \hat{S}_{j h 2}^{2}\right)}{n_{h}}+\sum_{h=1}^{L} \frac{W_{h}^{2} \hat{W}_{h 2} \hat{S}_{j h 2}^{2}}{r_{h}}=f_{0 j}(n, r)
$$

where $\bar{y}_{j(w)}$ is an unbiased estimate of the overall population mean $\bar{Y}_{j}$ of the $j^{\text {th }}$ characteristic and $V\left(\bar{y}_{j h(w)}\right)$ is as given in Equation (2).

Assuming a linear cost function the total cost $C$ of the sample survey may be given as:

$$
C=\sum_{h=1}^{L} c_{h 0} n_{h}+\sum_{h=1}^{L} c_{h 1} n_{h 1}+\sum_{h=1}^{L} c_{h 2} n_{h 2}
$$

where $c_{h 0}=$ the per unit cost of making the first attempt, $c_{h 1}=\sum_{h=1}^{L} c_{j h 1}$ is the per unit cost for processing the results of all the $p$ characteristics on the $n_{h 1}$ selected units from respondents group in the $h^{\text {th }}$ stratum in the first attempt and $c_{h 2}=\sum_{j=1}^{p} c_{j h 2}$ : the per unit cost for measuring and processing the results of all the $p$ characteristics on the $r_{h}$ units selected from the non respondents group in the $h^{\text {th }}$ stratum in the second attempt. Also, $c_{j h 1}$ and $c_{j h 2}$ are per unit costs of measuring the $j^{\text {th }}$ characteristic in first and second attempts respectively. As $n_{h 1}$ is not known until the first attempt has been made, the quantity $W_{h 1} n_{h 1}$ may be used as its expected value. The total expected cost $\hat{C}$ of the survey may be given as:

$$
\hat{C}=\sum_{h=1}^{L}\left(c_{h 0}+c_{h 1} W_{h 1}\right) n_{h}+\sum_{h=1}^{L} c_{h 2} r_{h}+\sum_{h=1}^{L} t_{h 0} \sqrt{n_{h}}+\sum_{h=1}^{L} t_{h 2} \sqrt{r_{h}}
$$

The problem therefore reduces to find the optimal values of sample sizes of respondents $n_{h}^{*}$ and nonrespondents $r_{h}^{*}$ which are expressed as:

$$
\left.\begin{array}{l}
\operatorname{Min}\left(f_{0 j}\right)=\sum_{h=1}^{L} \frac{W_{h}^{2}\left(S_{j h}^{2}-W_{h 2} S_{j h 2}^{2}\right)}{n_{h}}+\sum_{h=1}^{L} \frac{W_{h}^{2} W_{h 2} S_{j h 2}^{2}}{r_{h}} \\
\text { Subject to } \\
\quad \sum_{h=1}^{L}\left(c_{h 0}+c_{h 1} W_{h 1}\right) n_{h}+\sum_{h=1}^{L} c_{h 2} r_{h}+\sum_{h=1}^{L} t_{h 0} \sqrt{n_{h}}+\sum_{h=1}^{L} t_{h 2} \sqrt{r_{h}} \leq C_{0} \\
\quad n_{h}, r_{h} \geq 0 \text { and } h=1,2, \cdots, L
\end{array}\right\}, j=1,2, \cdots, p
$$

\section{Geometric Programming Formulation}

The following Multi-objective Nonlinear Programming Problem (MNLPP) with the cost function quadratic in $\sqrt{n_{h}}$ and significant travel cost is defined in Equations (6) as follows: 


$$
\left.\begin{array}{l}
\operatorname{Min} V\left(\hat{\pi}_{s j}\right)=\sum_{h=1}^{L} \frac{a_{h j}}{n_{h}} \\
\text { Subject to } \\
\sum_{h=1}^{L}\left(c_{h 0}+c_{h 1} W_{h 1}\right) n_{h}+\sum_{h=1}^{L} c_{h 2} r_{h}+\sum_{h=1}^{L} t_{h 0} \sqrt{n_{h}}+\sum_{h=1}^{L} t_{h 2} \sqrt{r_{h}} \leq C_{0} \\
\quad n_{h}, r_{h} \geq 0 \text { and } h=1,2, \cdots, L
\end{array}\right\} \quad j=1,2, \cdots, P
$$

Similarly, the expression (6) can be expressed in the standard Primal GPP with cost function quadratic in $\sqrt{n_{h}}$ where the travel cost is significant is given as follows:

$$
\left.\begin{array}{l}
\operatorname{Max} \quad f_{0 j}(n, r) \\
\text { Subject } f_{q}(n, r) \leq 1 \\
n_{h}, r_{h} \geq 0, h=1,2, \cdots, L
\end{array}\right\} j=1,2 \cdots, p
$$

where $f_{q}(n, r)=\sum_{i \in j[q]} d_{i}\left\{\left(n_{1}^{p_{i 1}} n_{2}^{p_{i 2}} \cdots n_{L}^{p_{i L}}\right)+\left(r_{1}^{p_{i 1}} r_{2}^{p_{i 2}} \cdots r_{L}^{p_{i L}}\right)\right\}, q=0,1,2, \cdots, k$

or $f_{q}(n, r)=\sum_{i \in j[q]} d_{i}\left\{\left[\prod_{h=1}^{L} n_{h}^{p_{i h}}\right]+\left[\prod_{h=1}^{L} r_{h}^{p_{\text {ih }}}\right]\right\}, d_{i}>0, n_{h}>0, q=0,1,2, \cdots, k$,

$p_{i h}$ : arbitrary real numbers, $d_{i}$ : positive and $f_{q}(n)$ : posinomials

Let for simplicity $f_{0 j}=a_{h j}$ and $d_{i}=a_{h j}=\left(c_{h 0}+c_{h 1} W_{h 1}\right) / C_{0}=c_{h 2} / C_{0}=t_{h 0} / C_{0}=t_{h 2} / C_{0}$

where $f_{q}(n, r)=\sum_{h=1}^{L} \frac{\left(c_{h 0}+c_{h 1} W_{h 1}\right)}{C_{0}} n_{h}+\sum_{h=1}^{L} \frac{c_{h 2}}{C_{0}} r_{h}+\sum_{h=1}^{L} \frac{t_{h 0}}{C_{0}} \sqrt{n_{h}}+\sum_{h=1}^{L} \frac{t_{h 2}}{C_{0}} \sqrt{r_{h}}$

The dual form of the Primal GPP which is stated in (7) can be given as:

$$
\begin{aligned}
& \operatorname{Max} v_{0 j}(w)=\prod_{q=0}^{k} \prod_{i \in j[q]}\left\{\left(\frac{d_{i}}{w_{i}}\right)^{w_{i}}\right\} \prod_{q=1}^{k}\left(\sum_{i \in j[q]} w_{i}\right)^{\sum_{i \in j q[]^{2}} w_{i}} \\
& \text { Subject to } \sum_{i \in[0]} w_{i}=1 \\
& \sum_{q=0}^{k} \sum_{i \in j[q]} p_{i h} w_{i}=0 \\
& w_{i} \geq 0, q=0,1, \cdots, k \text { and } i=1,2, \cdots, m_{k}
\end{aligned}
$$

The above formulated dual GPP (8) can be solved in the following two steps:

Step 1: For the Optimum value of the objective function, the objective function always takes the form:

$$
\begin{aligned}
& C_{0}\left(x^{*}\right)=\left(\frac{\text { Coeffi. of first term }}{w_{01}}\right)^{w_{01}} \times\left(\frac{\text { Coeffi. of Second term }}{w_{02}}\right)^{w_{02}} \\
& \times \cdots \times\left(\frac{\text { Coeffi. of last term }}{w_{k}}\right)^{w_{k}}\left(\sum w^{\prime} s \text { in the first constraint } s\right)^{\sum w^{\prime} s \text { in the first constraints }} \\
& \left(\sum w^{\prime} s \text { in the last constraint } s\right)^{\sum w^{\prime} s \text { in the last constraints }}
\end{aligned}
$$

The Multi-Objective objective function for our problem is:

$$
\prod_{q=0}^{k} \prod_{i \in j[q]}\left\{\left(\frac{d_{i}}{w_{i}}\right)^{w_{i}}\right\} \prod_{q=1}^{k}\left(\sum_{i \in j[q]} w_{i}\right)^{\sum_{i \in j[q]} w_{i}}
$$

Step 2: The equations that can be used for GPP for the weights are given below:

$\sum_{i \in j[0]} w_{i}$ in the objective function $=1$ (Normality condition ) and for each primal variable $n_{h} \& \sqrt{n_{h}}$ and $r_{h} \& \sqrt{r_{h}}$ having $m$ terms. 
$\sum_{i=1}^{m_{k}}\left(w_{i}\right.$ for each term $) \times\left(\right.$ exponent on $n_{h} \& \sqrt{n_{h}}$ and $r_{h} \& \sqrt{r_{h}}$ in that term $)=0$ (Orthogonality condition) and $w_{i} \geq 0$ (Positivity condition).

The above problem (8) has been solved with the help of steps (1-2) discussed in Section (3) and the corresponding solutions $w_{0 i}^{*}$ is the unique solution to the dual constraints; it will also maximize the objective function for the dual problem. Next, the solution of the primal problem will be obtained using primal-dual relationship theorem which is given below.

\section{Primal-Dual Relationship Theorem}

If $w_{0 i}^{*}$ is a maximizing point for dual problem (8), each minimizing points $\left(n_{1}, n_{2}, n_{3}, n_{4}\right.$ and $\left.r_{1}, r_{2}, r_{3}, r_{4}\right)$ for primal problem (7) satisfies the system of equations:

$$
f_{0 j}(n)= \begin{cases}w_{0 i}^{*} v\left(w^{*}\right), & i \in J[0], \\ \frac{w_{i j}}{v_{L}\left(w_{0 i}^{*}\right)}, & i \in J[L],\end{cases}
$$

where $L$ ranges over all positive integers for which $v_{L}\left(w_{0 i}^{*}\right)>0$.

The optimal values of sample sizes of the respondents $\left(n_{h}^{*}\right)$ and non-respondents $\left(r_{h}^{*}\right)$ can be calculated with the help of the primal-dual relationship theorem (9).

\section{Fuzzy Geometric Programming Approach}

The solution procedure to solve the problem (15) consists of the following steps:

Step-1: Solve the MOGPP as a single objective problem using only one objective at a time and ignoring the others. These solutions are known as ideal solution.

Step-2: From the results of step-1, determine the corresponding values for every objective at each solution derived. Let $\left(n^{(1)}, r^{(1)}\right),\left(n^{(2)}, r^{(2)}\right), \cdots,\left(n^{(j)}, r^{(j)}\right), \cdots,\left(n^{(p)}, r^{(p)}\right)$ are the ideal solutions of the objective functions $f_{01}\left(n^{(1)}, r^{(1)}\right), f_{02}\left(n^{(2)}, r^{(2)}\right), \cdots, f_{0 j}\left(n^{(j)}, r^{(j)}\right), \cdots, f_{0 p}\left(n^{(p)}, r^{(p)}\right)$.

So $U_{j}=\operatorname{Max}\left\{f_{01}\left(n^{(1)}, r^{(1)}\right), f_{02}\left(n^{(2)}, r^{(2)}\right), \cdots, f_{0 p}\left(n^{(p)}, r^{(p)}\right)\right\}$ and $L_{j}=f_{0 j}^{*}\left(n^{(j)}, r^{(j)}\right), j=1,2, \cdots, p$.

$\left[U_{j}\right.$ and $L_{j}$ be the upper and lower bonds of the $j^{\text {th }}$ objective function $f_{0 j}(n, r), j=1,2, \cdots, p$. $]$

Step 3: The membership function for the given problem can be define as:

$$
\mu_{j}\left(f_{0 j}(n, r)\right)= \begin{cases}0, & \text { if } f_{0 j}(n, r) \geq U_{j} \\ \frac{U_{j}(n, r)-f_{0 j}(n, r)}{U_{j}(n, r)-L_{j}(n, r)}, & \text { if } L_{j} \leq f_{0 j}(n, r) \geq U_{j}, j=1,2, \cdots, p \\ 1, & \text { if } f_{0 j}(n, r) \leq L_{j}\end{cases}
$$

Here $U_{j}(n, r)$ is a strictly monotonic decreasing function with respect to $f_{0 j}(n, r)$.

The membership functions in Equation (11)

i.e., $\mu_{j}\left(f_{0 j}(n, r)\right), j=1,2, \cdots, p$

Therefore the general aggregation function can be defined as

$\mu_{\tilde{D}}(n, r)=\mu_{\tilde{D}}\left\{\mu_{1}\left(f_{01}(n, r)\right), \mu_{2}\left(f_{02}(n, r)\right), \cdots, \mu_{p}\left(f_{0 p}(n, r)\right)\right\}$

The fuzzy multi-objective formulation of the problem can be defined as:

$$
\left.\begin{array}{l}
\operatorname{Max} \mu_{\tilde{D}}(n, r) \\
\text { Subject to } \\
\sum_{h=1}^{L} \frac{\left(c_{h 0}+c_{h 1} W_{h 1}\right)}{C_{0}} n_{h}+\sum_{h=1}^{L} \frac{C_{h 2}}{C_{0}} r_{h}+\sum_{h=1}^{L} \frac{t_{h 0}}{C_{0}} \sqrt{n_{h}}+\sum_{h=1}^{L} \frac{t_{h 2}}{C_{0}} \sqrt{r_{h}} \leq 1 ; \\
n_{h}, r_{h} \geq 0 \text { and } j=1,2, \cdots, p .
\end{array}\right\}
$$


The problem to find the optimal values of $\left(n^{*}, r^{*}\right)$ for this convex-fuzzy decision based on addition operator (like Tiwari et. al. [29]). Therefore the problem (11) is reduced according to max-addition operator as:

$$
\left.\begin{array}{c}
\operatorname{Max} \mu_{D}\left(n^{*}, r^{*}\right)=\sum_{j=1}^{p} \mu_{j}\left(f_{0 j}(n, r)\right)=\sum_{j=1}^{p} \frac{U_{j}-\left(f_{0 j}(n, r)\right)}{U_{j}-L_{j}} \\
\text { Subject to } \quad f_{q}(n, r) \leq 1 ; \\
0 \leq \mu_{j}\left(f_{0 j}(n, r)\right) \leq 1 \\
n_{h}, r_{h} \geq 0 \text { and } j=1,2, \cdots, p .
\end{array}\right\}
$$

The above problem (12) reduces to

$$
\begin{aligned}
& \operatorname{Max} \mu_{D}\left(n^{*}, r^{*}\right)=\sum_{j=1}^{p}\left\{\frac{U_{j}}{U_{j}-L_{j}}-\frac{\left(f_{0 j}(n, r)\right)}{U_{j}-L_{j}}\right\} \\
& \text { Subject to } \\
& \left.\qquad \begin{array}{l}
f_{q}(n, r) \leq 1 ; \\
n_{h}, r_{h} \geq 0 \text { and } j=1,2, \cdots, p .
\end{array}\right\}
\end{aligned}
$$

The problem (13) maximizes if the function $F_{o j}(n)=\left\{\frac{\left(f_{0 j}(n, r)\right)}{U_{j}-L_{j}}\right\}$ attain the minimum values. Therefore the problem (13) reduces into the primal problem (14) define as:

$$
\left.\begin{array}{l}
\operatorname{Min} \sum_{j=0}^{p} F_{o j}(n, r) \\
\text { Subject to } \\
f_{q}(n, r) \leq 1 ; \\
n_{h}, r_{h} \geq 0 \text { and } h=1,2, \cdots, L
\end{array}\right\}
$$

The dual form of the Primal GPP which is stated in (16) can be given as:

$$
\begin{gathered}
\text { Max } v(w)=\prod_{q=0}^{k} \prod_{i \in j[q]}\left\{\left(\frac{d_{i}}{w_{i}}\right)^{w_{i}}\right\} \prod_{q=1}^{k}\left(\sum_{i \in j[q]} w_{i}\right)^{\sum_{i \in j[q]} w_{i}} \\
\text { Subject } \sum_{i \in[0]} w_{i}=1 \\
\sum_{q=0}^{k} \sum_{i \in j[q]} p_{i h} w_{i}=0 \\
w_{i} \geq 0, q=0,1, \cdots, k \text { and } i=1,2, \cdots, m_{k}
\end{gathered}
$$

The optimal values of sample sizes of the respondents $\left(n_{h}^{*}\right)$ and non-respondents $\left(r_{h}^{*}\right)$ can be calculated with the help of the primal-dual relationship theorem (9).

\section{Numerical}

In Table 1, the stratum sizes, stratum weights, stratum standard deviations, measurement costs and the travel costs within the stratum are given for two characteristics under study in a population stratified in four strata. The data are mainly from Khan et al. [8]. The travelling costs $t_{h 0}$ and $t_{h 2}$ are assumed.

The total budget available for the survey is taken as $C_{0}=5000$. The relative values of the variances of the non-respondents and respondents, that is $S_{j h 2}^{2} / S_{j h}^{2}$ is assumed to be constant and equal to 0.25 for $j=1,2$ and $h$ $=1,2,3,4$. However, these ratios may vary from stratum to stratum and from characteristic to characteristic and can be handled accordingly.

For solving MOGPP by using fuzzy programming, we shall first solve the two sub-problems: 
Table 1. Data for four Strata and two characteristics.

\begin{tabular}{ccccccccccc}
\hline$h$ & $N_{h}$ & $S_{1 h}^{2}$ & $S_{2 h}^{2}$ & $w_{h 1}$ & $w_{h 2}$ & $c_{h 0}$ & $c_{h 1}$ & $c_{h 2}$ & $t_{h 0}$ & $t_{h 2}$ \\
\hline 1 & 1214 & 4817.72 & 8121.15 & 0.7 & 0.30 & 1 & 2 & 3 & 0.5 & 2 \\
2 & 822 & 6251.26 & 7613.52 & 0.80 & 0.20 & 1 & 3 & 4 & 0.5 & 2.5 \\
3 & 1028 & 3066.16 & 1456.4 & 0.75 & 0.25 & 1 & 4 & 5 & 0.5 & 3 \\
4 & 786 & 6207.25 & 6977.72 & 0.72 & 0.28 & 1 & 5 & 6 & 0.5 & 4.5 \\
\hline
\end{tabular}

Sub problem 1: On substituting the table values in sub-problem 1, we have obtained the expressions given below:

$$
\begin{aligned}
& \text { Min } f_{01}=\frac{456.3344}{n_{1}}+\frac{261.8965}{n_{2}}+\frac{209.5529}{n_{3}}+\frac{230.9097}{n_{4}} \\
& \quad+\frac{11.10002688}{r_{1}}+\frac{2.75680566}{r_{2}}+\frac{3.492547875}{r_{3}}+\frac{4.866484}{r_{4}} \\
& \text { Subject to } \\
& \begin{array}{l}
0.00048 n_{1}+0.00068 n_{2}+0.0008 n_{3}+0.00092 n_{4} \\
+0.0006 r_{1}+0.0008 r_{2}+0.001 r_{3}+0.0012 r_{4}
\end{array} \\
& +0.0001 \sqrt{n_{1}}+0.0001 \sqrt{n_{2}}+0.0001 \sqrt{n_{3}}+0.0001 \sqrt{n_{4}} \\
& +0.0004 \sqrt{r_{1}}+0.0005 \sqrt{r_{2}}+0.0006 \sqrt{r_{3}}+0.0009 \sqrt{r_{4}} \leq 1 \\
& n_{h} \geq 0, r_{h} \geq 0, n_{h}, r_{h} \text { areintegers; } h=1,2, \cdots, L
\end{aligned}
$$

The dual of the above problem (16) is obtained as:

$$
\begin{aligned}
& \operatorname{Max} v\left(w_{0 i}^{*}\right)=\left(\left(456.3344 / w_{01}\right)^{w_{01}}\right) \times\left(\left(261.8965 / w_{02}\right)^{w_{02}}\right) \times\left(\left(209.5529 / w_{03}\right)^{w_{03}}\right) \times\left(\left(230.9097 / w_{04}\right)^{w_{04}}\right) \\
& \times\left(\left(11.100027 / w_{05}\right)^{w_{05}}\right) \times\left(\left(2.756806 / w_{06}\right)^{w_{06}}\right) \times\left(\left(3.492548 / w_{07}\right)^{w_{07}}\right) \times\left(\left(4.866484 / w_{08}\right)^{w_{08}}\right) \\
& \times\left(\left(\frac{0.00048}{w_{11}}\right)^{w_{11}}\right) \times\left(\left(\frac{0.00068}{w_{12}}\right)^{w_{12}}\right) \times\left(\left(\frac{0.0008}{w_{13}}\right)^{w_{13}}\right) \times\left(\left(\frac{0.00092}{w_{14}}\right)^{w_{14}}\right) \times\left(\left(\frac{0.0006}{w_{15}}\right)^{w_{15}}\right) \\
& \times\left(\left(\frac{0.0008}{w_{16}}\right)^{w_{16}}\right) \times\left(\left(\frac{0.001}{w_{17}}\right)^{w_{17}}\right) \times\left(\left(\frac{0.0012}{w_{18}}\right)^{w_{18}}\right) \times\left(\left(\frac{0.0001}{w_{19}}\right)^{w_{19}}\right) \times\left(\left(\frac{0.0001}{w_{20}}\right)^{w_{20}}\right) \times\left(\left(\frac{0.0001}{w_{21}}\right)^{w_{21}}\right) \\
& \times\left(\left(\frac{0.0001}{w_{22}}\right)^{w_{22}}\right) \times\left(\left(\frac{0.0004}{w_{23}}\right)^{w_{23}}\right) \times\left(\left(\frac{0.0005}{w_{24}}\right)^{w_{24}}\right) \times\left(\left(\frac{0.0006}{w_{25}}\right)^{w_{25}}\right) \times\left(\left(\frac{0.0009}{w_{26}}\right)^{w_{26}}\right) \\
& \times\left(\left(w_{11}+w_{12}+w_{13}+w_{14}+w_{15}+w_{16}+w_{17}+w_{18}+w_{19}+w_{20}+w_{21}+w_{22}+w_{23}+w_{24}+w_{25}+w_{26}\right) \wedge\right. \\
& \left.\left(w_{11}+w_{12}+w_{13}+w_{14}+w_{15}+w_{16}+w_{17}+w_{18}+w_{19}+w_{20}+w_{21}+w_{22}+w_{23}+w_{24}+w_{25}+w_{26}\right)\right) \text {; } \\
& \text { Subject to } w_{01}+w_{02}+w_{03}+w_{04}+w_{05}+w_{06}+w_{07}+w_{08}=1 ; \text { (normality condition) } \\
& -w_{01}+w_{11}+(1 / 2) w_{19}=0 \\
& -w_{02}+w_{12}+(1 / 2) w_{20}=0 \\
& -w_{03}+w_{13}+(1 / 2) w_{21}=0 \\
& -w_{04}+w_{14}+(1 / 2) w_{22}=0 \\
& \left.-w_{05}+w_{15}+(1 / 2) w_{23}=0\right\} \text { (orthogonality condition) } \\
& -w_{06}+w_{16}+(1 / 2) w_{24}=0 \\
& -w_{07}+w_{17}+(1 / 2) w_{25}=0 \\
& -w_{08}+w_{18}+(1 / 2) w_{26}=0
\end{aligned}
$$


For orthogonality condition defined in expression 17(iii) are evaluated with the help of the payoff matrix which is defined below

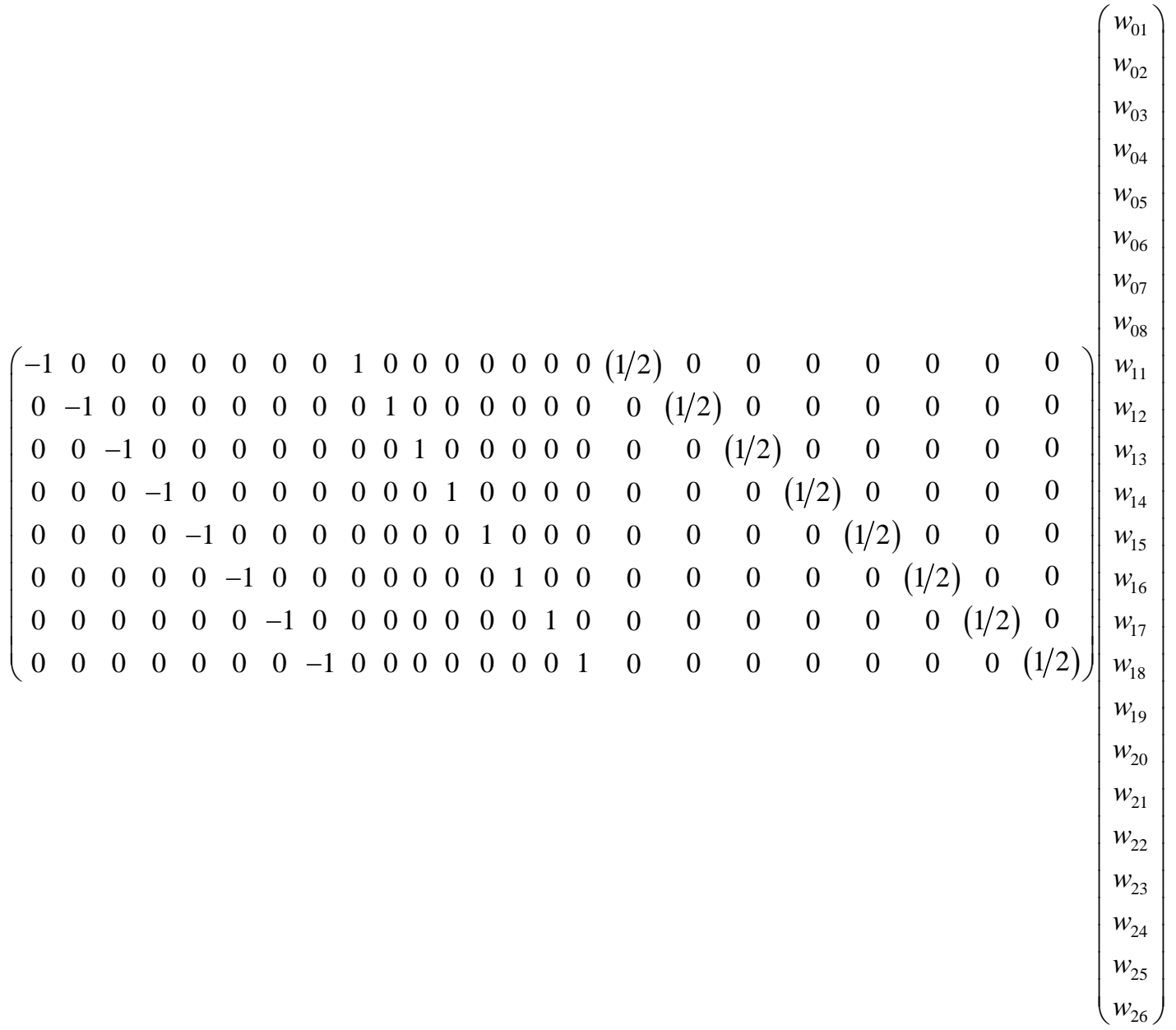$$
\Rightarrow
$$

$$
\begin{aligned}
& -w_{01}+w_{11}+(1 / 2) w_{19}=0 \\
& -w_{02}+w_{12}+(1 / 2) w_{20}=0 \\
& -w_{03}+w_{13}+(1 / 2) w_{21}=0 \\
& -w_{04}+w_{14}+(1 / 2) w_{22}=0 \\
& -w_{05}+w_{15}+(1 / 2) w_{23}=0 \\
& -w_{06}+w_{16}+(1 / 2) w_{24}=0 \\
& -w_{07}+w_{17}+(1 / 2) w_{25}=0 \\
& -w_{08}+w_{18}+(1 / 2) w_{26}=0
\end{aligned}
$$

Solving the above formulated dual problem (17) with the help of Lingo software, we have the corresponding dual solutions as follows:

$$
\begin{aligned}
& w_{01}=0.2306558, w_{02}=0.2079228, w_{03}=0.2017047, w_{04}=0.2270049, w_{05}=0.04094539, \\
& w_{06}=0.02376402, w_{07}=0.02986826, w_{08}=0.03813407, \text { and } v\left(w^{*}\right)=4.175039 .
\end{aligned}
$$

Using the primal dual-relationship theorem (9), we have the optimal solution of primal problem: i.e., the optimal sample sizes of respondents and non respondents are computed as follows:

$$
f_{0 j}(n, r)=w_{0 i}^{*} v\left(w_{0 i}^{*}\right)
$$

In expression (16), we first keep the $r$ constant and calculate the values of $n$ as: 


$$
\begin{array}{ll}
f_{01}\left(n_{1}, r\right)=w_{01}^{*} v\left(w_{0 i}^{*}\right) \Rightarrow n_{1} \cong 474 & f_{02}\left(n_{2}, r\right)=w_{02}^{*} v\left(w_{0 i}^{*}\right) \Rightarrow n_{2} \cong 302 \\
f_{03}\left(n_{3}, r\right)=w_{03}^{*} v\left(w_{0 i}^{*}\right) \Rightarrow n_{3} \cong 249 & f_{04}\left(n_{4}, r\right)=w_{04}^{*} v\left(w_{0 i}^{*}\right) \Rightarrow n_{4} \cong 244
\end{array}
$$

Now, from the expression (16), we keep the $n$ constant and calculate the values of $r$ as:

$$
\begin{array}{ll}
f_{01}\left(n, r_{1}\right)=w_{01}^{*} v\left(w_{0 i}^{*}\right) \Rightarrow r_{1} \cong 65 & f_{02}\left(n, r_{2}\right)=w_{02}^{*} v\left(w_{0 i}^{*}\right) \Rightarrow r_{2} \cong 28 \\
f_{03}\left(n, r_{3}\right)=w_{03}^{*} v\left(w_{0 i}^{*}\right) \Rightarrow r_{3} \cong 28 & f_{04}\left(n, r_{4}\right)=w_{04}^{*} v\left(w_{0 i}^{*}\right) \Rightarrow r_{4} \cong 31
\end{array}
$$

The optimal values and the objective function value are given below:

$n_{1}^{*}=474, n_{2}^{*}=302, n_{3}^{*}=249$ and $n_{4}^{*}=244$;

$r_{1}^{*}=65, r_{2}^{*}=28, r_{3}^{*}=28$ and $r_{4}^{*}=31$ and theobjective value of the primal problem is 4.175039 .

Sub problem 2: On substituting the table values in sub-problem 2, we have obtained the expressions given below:

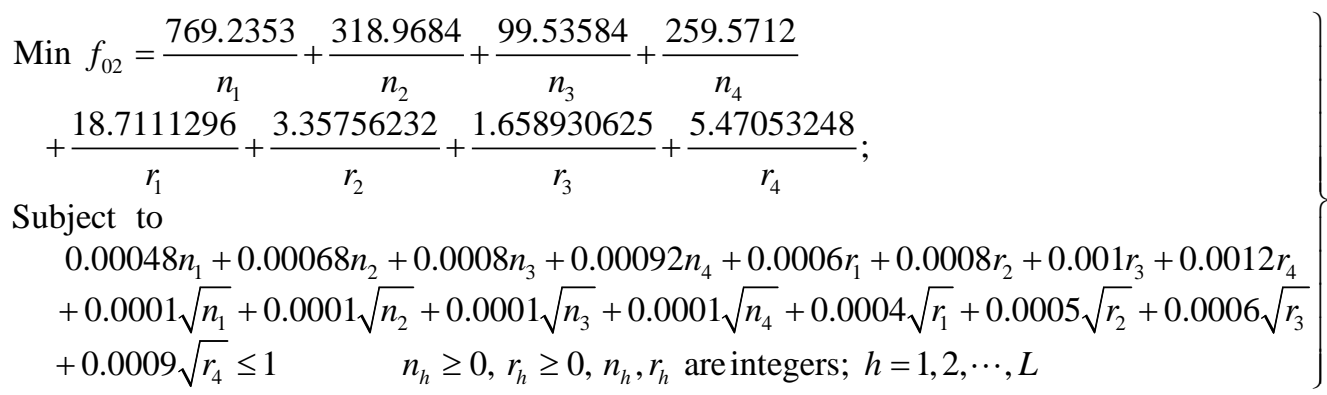

The dual of the above problem (18) is obtained as follows:

$$
\begin{aligned}
& \operatorname{Max} v\left(w_{0 i}^{*}\right)=\left(\left(769.2353 / w_{01}\right)^{w_{01}}\right) \times\left(\left(318.9684 / w_{02}\right)^{w_{02}}\right) \times\left(\left(99.53584 / w_{03}\right)^{w_{03}}\right) \times\left(\left(259.5712 / w_{04}\right)^{w_{04}}\right) \\
& \times\left(\left(18.7111296 / w_{05}\right)^{w_{05}}\right) \times\left(\left(3.35756232 / w_{06}\right)^{w_{06}}\right) \times\left(\left(1.658930625 / w_{07}\right)^{w_{07}}\right) \times\left(\left(5.47053248 / w_{08}\right)^{w_{08}}\right) \\
& \times\left(\left(\frac{0.00048}{w_{11}}\right)^{w_{11}}\right) \times\left(\left(\frac{0.00068}{w_{12}}\right)^{w_{12}}\right) \times\left(\left(\frac{0.0008}{w_{13}}\right)^{w_{13}}\right) \times\left(\left(\frac{0.00092}{w_{14}}\right)^{w_{14}}\right) \times\left(\left(\frac{0.0006}{w_{15}}\right)^{w_{15}}\right) \times\left(\left(\frac{0.0008}{w_{16}}\right)^{w_{16}}\right) \\
& \times\left(\left(\frac{0.001}{w_{17}}\right)^{w_{17}}\right) \times\left(\left(\frac{0.0012}{w_{18}}\right)^{w_{18}}\right) \times\left(\left(\frac{0.0001}{w_{19}}\right)^{w_{19}}\right) \times\left(\left(\frac{0.0001}{w_{20}}\right)^{w_{20}}\right) \times\left(\left(\frac{0.0001}{w_{21}}\right)^{w_{21}}\right) \times\left(\left(\frac{0.0001}{w_{22}}\right)^{w_{22}}\right) \\
& \times\left(\left(\frac{0.0004}{w_{23}}\right)^{w_{23}}\right) \times\left(\left(\frac{0.0005}{w_{24}}\right)^{w_{24}}\right) \times\left(\left(\frac{0.0006}{w_{25}}\right)^{w_{25}}\right) \times\left(\left(\frac{0.0009}{w_{26}}\right)^{w_{26}}\right) \\
& \times\left(\left(w_{11}+w_{12}+w_{13}+w_{14}+w_{15}+w_{16}+w_{17}+w_{18}+w_{19}+w_{20}+w_{21}+w_{22}+w_{23}+w_{24}+w_{25}+w_{26}\right)^{\wedge}\right. \\
& \left.\left(w_{11}+w_{12}+w_{13}+w_{14}+w_{15}+w_{16}+w_{17}+w_{18}+w_{19}+w_{20}+w_{21}+w_{22}+w_{23}+w_{24}+w_{25}+w_{26}\right)\right) ; \\
& \text { Subject to } \\
& w_{01}+w_{02}+w_{03}+w_{04}+w_{05}+w_{06}+w_{07}+w_{08}=1 ; \text { (normality condition) } \\
& -w_{01}+w_{11}+(1 / 2) w_{19}=0 \\
& -w_{02}+w_{12}+(1 / 2) w_{20}=0 \\
& -w_{03}+w_{13}+(1 / 2) w_{21}=0 \\
& -w_{04}+w_{14}+(1 / 2) w_{22}=0 \\
& -w_{05}+w_{15}+(1 / 2) w_{23}=0 \\
& -w_{06}+w_{16}+(1 / 2) w_{24}=0 \\
& -w_{07}+w_{17}+(1 / 2) w_{25}=0 \\
& -w_{08}+w_{18}+(1 / 2) w_{26}=0
\end{aligned}
$$


For orthogonality condition defined in expression 19(iii) are evaluated with the help of the payoff matrix which is defined below:

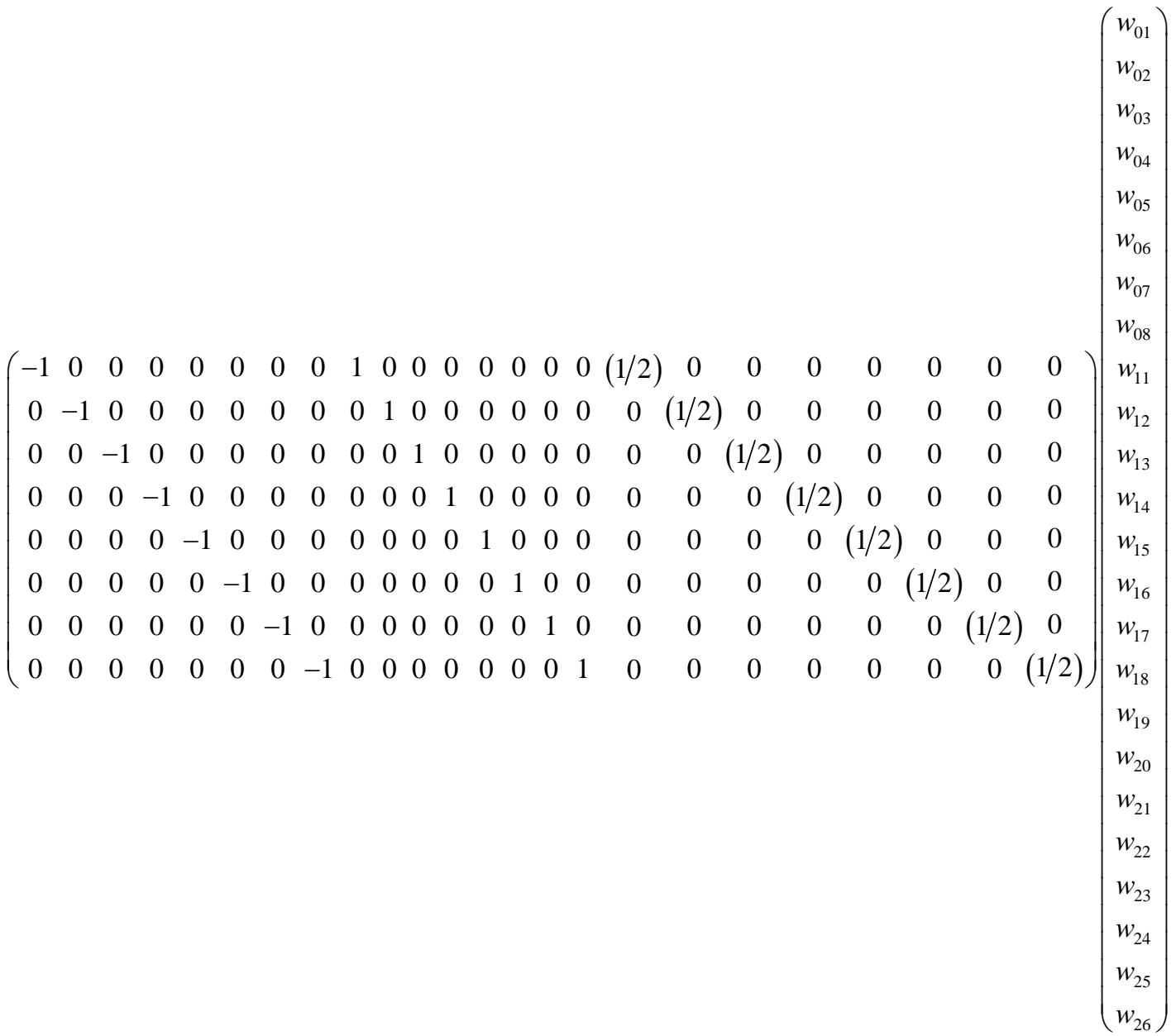

$$
\begin{aligned}
& \Rightarrow \\
& -w_{01}+w_{11}+(1 / 2) w_{19}=0 \\
& -w_{02}+w_{12}+(1 / 2) w_{20}=0 \\
& -w_{03}+w_{13}+(1 / 2) w_{21}=0 \\
& -w_{04}+w_{14}+(1 / 2) w_{22}=0 \\
& -w_{05}+w_{15}+(1 / 2) w_{23}=0 \\
& -w_{06}+w_{16}+(1 / 2) w_{24}=0 \\
& -w_{07}+w_{17}+(1 / 2) w_{25}=0 \\
& -w_{08}+w_{18}+(1 / 2) w_{26}=0
\end{aligned}
$$

Solving the above formulated dual problems, we have the corresponding solution as:

$w_{01}=0.2854095, w_{02}=0.2187303, w_{03}=0.1325806, w_{04}=0.2294348, w_{05}=0.04989059$,

$w_{06}=0.05057448, w_{07}=0.02498275, w_{08}=0.01974803$, and $v\left(w^{*}\right)=4.593918$.

The optimal values of sample sizes of respondents and non-respondents $\left(n_{h}^{*}, r_{h}^{*}\right)$ can be calculated with the 
help of the primal-dual relationship theorem (9) as we have calculated in the sub-problem 1 are given as follows:

$n_{1}^{*}=587, n_{2}^{*}=317, n_{3}^{*}=163$ and $n_{4}^{*}=246 ;$

$r_{1}^{*}=81, r_{2}^{*}=29, r_{3}^{*}=18$ and $r_{4}^{*}=31$ and theobjective value of the primal problem is 4.593918 .

Now the pay-off matrix of the above problems is given below:

\begin{tabular}{|c|c|c|}
\hline & $f_{01}(n, r)$ & $f_{02}(n, r)$ \\
\hline$\left(n^{(1)}, r^{(1)}\right)$ & [4.175039 & 4.793168 \\
\hline$\left(n^{(2)}, r^{(2)}\right)$ & 4.403294 & 4.593918 \\
\hline
\end{tabular}

The lower and upper bond of $f_{01}(n, r)$ and $f_{02}(n, r)$ can be obtained from the pay-off matrix

$4.175039 \leq f_{01}(n, r) \leq 4.403294$ and $4.593918 \leq f_{02}(n, r) \leq 4.793168$.

Let $\mu_{1}(n, r)$ and $\mu_{2}(n, r)$ be the fuzzy membership function of the objective function $f_{01}(n, r)$ and $f_{02}(n, r)$ respectively and they are defined as:

$$
\begin{aligned}
& \mu_{1}(n, r)= \begin{cases}1, & \text { if } f_{01}(n, r) \leq 4.175039 \\
\frac{4.403294-f_{01}(n, r)}{0.228255}, & \text { if } 4.175039 \leq f_{01}(n, r) \leq 4.403294 \\
0, & \text { if } f_{01}(n, r) \geq 4.403294\end{cases} \\
& \mu_{2}(n, r)= \begin{cases}1, & \text { if } Z_{2}(n, r) \leq 4.593918 \\
\frac{4.793168-Z_{2}(n, r)}{0.19925}, & \text { if } 4.593918 \leq Z_{2}(n, r) \leq 4.793168 \\
0, & \text { if } Z_{2}(n, r) \geq 4.793168\end{cases}
\end{aligned}
$$

On applying the max-addition operator, the MOGPP, the standard primal problem reduces to the problem as:

$$
\begin{aligned}
& \text { Maximize }\left\{43.3472-\left(\frac{f_{01}(n, r)}{0.228255}+\frac{f_{02}(n, r)}{0.19925}\right)\right\} \\
& \text { Subject to } \\
& \quad 0.00048 n_{1}+0.00068 n_{2}+0.0008 n_{3}+0.00092 n_{4} \\
& \quad+0.0006 r_{1}+0.0008 r_{2}+0.001 r_{3}+0.0012 r_{4} \\
& \quad+0.0001 \sqrt{n_{1}}+0.0001 \sqrt{n_{2}}+0.0001 \sqrt{n_{3}}+0.0001 \sqrt{n_{4}} \\
& \quad+0.0004 \sqrt{r_{1}}+0.0005 \sqrt{r_{2}}+0.0006 \sqrt{r_{3}}+0.0009 \sqrt{r_{4}} \leq 1 \\
& \quad n_{h} \geq 0, r_{h} \geq 0, n_{h}, r_{h} \text { areintegers; } h=1,2, \cdots, L
\end{aligned}
$$

In order to maximize the above problem, we have to minimize $\left(\frac{f_{01}(n, r)}{0.228255}+\frac{f_{02}(n, r)}{0.19925}\right)$, subject to the constraints as described below: 


$$
\begin{gathered}
\operatorname{Min}\left\{\begin{array}{l}
\frac{5859.8847}{n_{1}}+\frac{2748.2334}{n_{2}}+\frac{1417.6227}{n_{3}}+\frac{2314.3744}{n_{4}} \\
+\frac{142.5377}{r_{1}}+\frac{28.9287}{r_{2}}+\frac{23.627}{r_{3}}+\frac{48.7761}{r_{4}}
\end{array}\right\} \\
\text { Subject to } 0.00048 n_{1}+0.00068 n_{2}+0.0008 n_{3}+0.00092 n_{4} \\
\quad+0.0006 r_{1}+0.0008 r_{2}+0.001 r_{3}+0.0012 r_{4} \\
+0.0001 \sqrt{n_{1}}+0.0001 \sqrt{n_{2}}+0.0001 \sqrt{n_{3}}+0.0001 \sqrt{n_{4}} \\
+0.0004 \sqrt{r_{1}}+0.0005 \sqrt{r_{2}}+0.0006 \sqrt{r_{3}}+0.0009 \sqrt{r_{4}} \leq 1 \\
\quad n_{h} \geq 0, r_{h} \geq 0, n_{h}, r_{h} \text { areintegers; } h=1,2, \cdots, L
\end{gathered}
$$

Degree of Difficulty of the problem $(21)$ is $=(24-(8+1)=15$.

Hence the dual problem of the above final formulated problem (21) is given as:

$\left.\operatorname{Max} v\left(w_{0 i}^{*}\right)=\left(\left(5859.8847 / w_{01}\right)^{w_{01}}\right) \times\left(\left(2748.2334 / w_{02}\right)^{w_{02}}\right) \times\left(\left(1417.6227 / w_{03}\right)^{w_{03}}\right) \times\left(\left(2314.3744 / w_{04}\right)^{w_{04}}\right)\right)$ $\times\left(\left(142.5377 / w_{05}\right)^{w_{05}}\right) \times\left(\left(28.9287 / w_{06}\right)^{w_{06}}\right) \times\left(\left(23.627 / w_{07}\right)^{w_{07}}\right) \times\left(\left(48.7761 / w_{08}\right)^{w_{08}}\right)$

$\times\left(\left(\frac{0.00048}{w_{11}}\right)^{w_{11}}\right) \times\left(\left(\frac{0.00068}{w_{12}}\right)^{w_{12}}\right) \times\left(\left(\frac{0.0008}{w_{13}}\right)^{w_{13}}\right) \times\left(\left(\frac{0.00092}{w_{14}}\right)^{w_{14}}\right) \times\left(\left(\frac{0.0006}{w_{15}}\right)^{w_{15}}\right) \times\left(\left(\frac{0.0008}{w_{16}}\right)^{w_{16}}\right)$

$\times\left(\left(\frac{0.001}{w_{17}}\right)^{w_{17}}\right) \times\left(\left(\frac{0.0012}{w_{18}}\right)^{w_{18}}\right) \times\left(\left(\frac{0.0001}{w_{19}}\right)^{w_{19}}\right) \times\left(\left(\frac{0.0001}{w_{20}}\right)^{w_{20}}\right) \times\left(\left(\frac{0.0001}{w_{21}}\right)^{w_{21}}\right) \times\left(\left(\frac{0.0001}{w_{22}}\right)^{w_{22}}\right)$

$\times\left(\left(\frac{0.0004}{w_{23}}\right)^{w_{23}}\right) \times\left(\left(\frac{0.0005}{w_{24}}\right)^{w_{24}}\right) \times\left(\left(\frac{0.0006}{w_{25}}\right)^{w_{25}}\right) \times\left(\left(\frac{0.0009}{w_{26}}\right)^{w_{26}}\right)$

$\times\left(\left(w_{11}+w_{12}+w_{13}+w_{14}+w_{15}+w_{16}+w_{17}+w_{18}+w_{19}+w_{20}+w_{21}+w_{22}+w_{23}+w_{24}+w_{25}+w_{26}\right) \wedge\right.$

$\left.\left(w_{11}+w_{12}+w_{13}+w_{14}+w_{15}+w_{16}+w_{17}+w_{18}+w_{19}+w_{20}+w_{21}+w_{22}+w_{23}+w_{24}+w_{25}+w_{26}\right)\right)$;

Subject to $\quad w_{01}+w_{02}+w_{03}+w_{04}+w_{05}+w_{06}+w_{07}+w_{08}=1$; (normality condition)

$$
\left.\begin{array}{r}
-w_{01}+w_{11}+(1 / 2) w_{19}=0 \\
-w_{02}+w_{12}+(1 / 2) w_{20}=0 \\
-w_{03}+w_{13}+(1 / 2) w_{21}=0 \\
-w_{04}+w_{14}+(1 / 2) w_{22}=0 \\
-w_{05}+w_{15}+(1 / 2) w_{23}=0 \\
-w_{06}+w_{16}+(1 / 2) w_{24}=0 \\
-w_{07}+w_{17}+(1 / 2) w_{25}=0 \\
-w_{08}+w_{18}+(1 / 2) w_{26}=0
\end{array}\right\}
$$

$\left.\begin{array}{l}w_{01}, w_{02}, w_{03}, w_{04}, w_{05}, w_{06}, w_{07}, w_{08}>0 ; \\ w_{11}, w_{12}, w_{13}, w_{14}, w_{15}, w_{16}, w_{17}, w_{18}, w_{19}, w_{20}, w_{21}, w_{22}, w_{23}, w_{24}, w_{25}, w_{26} \geq 0\end{array}\right\}$ (positivity condition) (iv)

For orthogonality condition defined in expression 22(iii) are evaluated with the help of the payoff matrix which is defined below: 


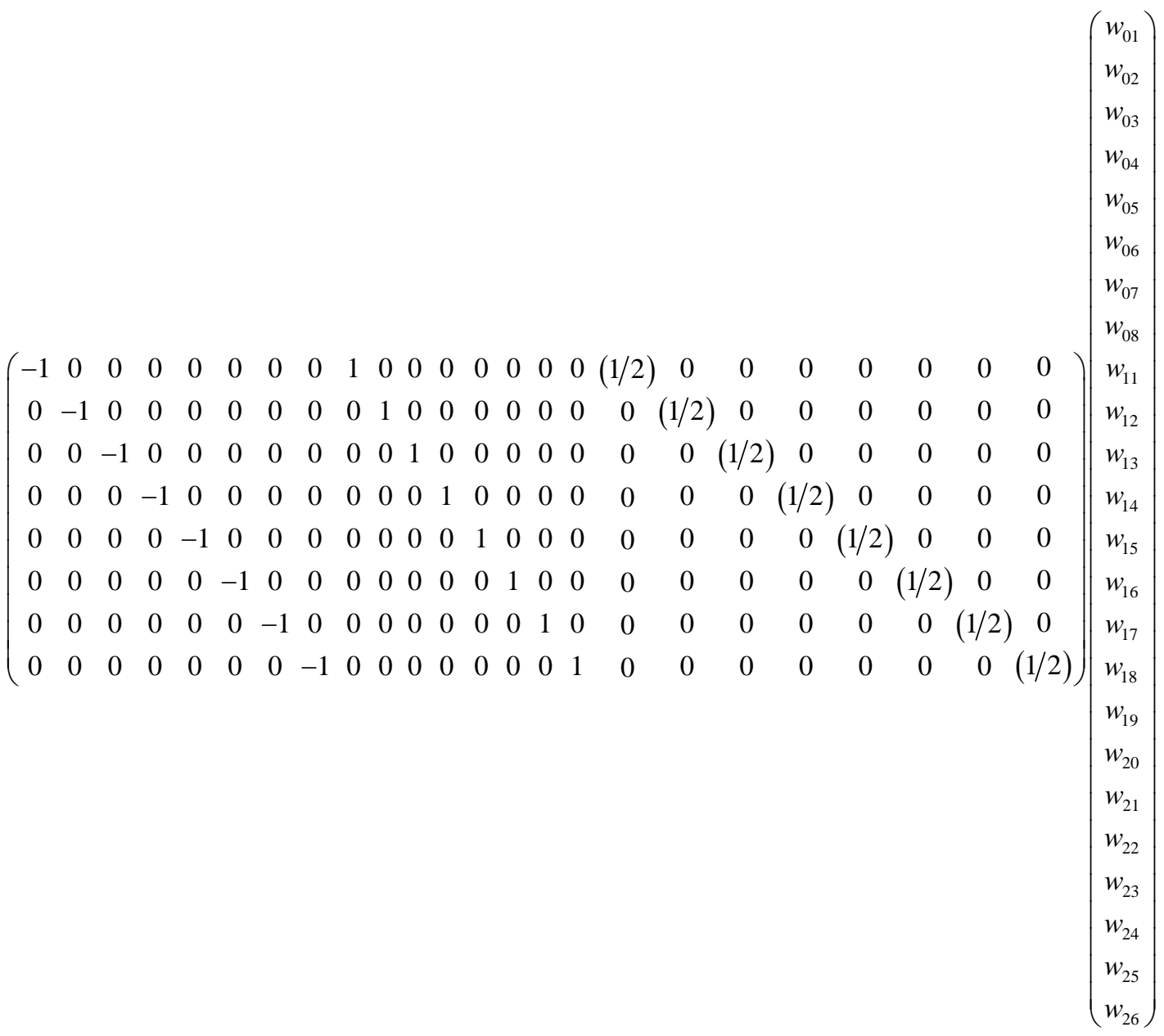

$$
\begin{aligned}
& \Rightarrow \\
& -w_{01}+w_{11}+(1 / 2) w_{19}=0 \\
& -w_{02}+w_{12}+(1 / 2) w_{20}=0 \\
& -w_{03}+w_{13}+(1 / 2) w_{21}=0 \\
& -w_{04}+w_{14}+(1 / 2) w_{22}=0 \\
& -w_{05}+w_{15}+(1 / 2) w_{23}=0 \\
& -w_{06}+w_{16}+(1 / 2) w_{24}=0 \\
& -w_{07}+w_{17}+(1 / 2) w_{25}=0 \\
& -w_{08}+w_{18}+(1 / 2) w_{26}=0
\end{aligned}
$$

After solving the formulated dual problem (22) using lingo software we obtain the following values of the dual variables which are given as:

$$
\begin{aligned}
& w_{01}=0.2610739, w_{02}=0.2127708, w_{03}=0.1657664, w_{04}=0.2270332, w_{05}=0.04629584, \\
& w_{06}=0.02431073, w_{07}=0.02461037, w_{08}=0.03813880, \text { and } v\left(w^{*}\right)=41.83433 .
\end{aligned}
$$

The optimal values of sample sizes of respondents and non-respondents $\left(n_{h}^{*}, r_{h}^{*}\right)$ can be calculated with the help of the primal-dual relationship theorem (9) as we have calculated in the sub-problem 1 are given as follows: 
$n_{1}^{*}=537, n_{2}^{*}=309, n_{3}^{*}=204$ and $n_{4}^{*}=244 ; r_{1}^{*}=74, r_{2}^{*}=28, r_{3}^{*}=23$ and $r_{4}^{*}=31$

and the objective value of the primal problem is 41.83433 .

\section{Conclusion}

This paper provides an insightful study of fuzzy programming for solving the multi-objective geometric programming problem (MOGPP). The problem of non-response with significant travel costs where the cost is quadratic in $\sqrt{n_{h}}$ in multivariate stratified sample surveys has been formulated of as a Multi-Objective Geometric Programming Problem (MOGPP). The fuzzy programming approach has described for solving the formulated MOGPP. The formulated MOGPP has been solved with the help of LINGO Software [30] and the dual solution is obtained. The optimum allocations of sample sizes of respondents and non respondents are obtained with the help of dual solutions and primal-dual relationship theorem. To ascertain the practical utility of the proposed method in sample surveys problem in presence of non-response with significant travel cost where the cost is quadratic in $\sqrt{n_{h}}$, a numerical example is also given to illustrate the procedure.

\section{References}

[1] Cochran, W.G. (1977) Sampling Techniques. 3rd Edition, John Wiley and Sons, New York.

[2] Sukhatme, P.V., Sukhatme, B.V., Sukhatme, S. and Asok, C. (1984) Sampling Theory of Surveys with Applications. Iowa State University Press, Ames and Indian Society of Agricultural Statistics, New Delhi.

[3] Hansen, M.H. and Hurwitz, W.N. (1946) The Problem of Non-Response in Sample Surveys. Journal of the American Statistical Association, 41, 517-529. http://dx.doi.org/10.1080/01621459.1946.10501894

[4] El-Badry, M.A. (1956) A Sampling Procedure for Mailed Questionnaires. Journal of the American Statistical Association, 51, 209-227. http://dx.doi.org/10.1080/01621459.1956.10501321

[5] Fordori, G.T. (1961) Some Non-Response Sampling Theory for Two Stage Designs. Mimeograph Series No. 297, North Carolina State University, Raleigh.

[6] Srinath, K.P. (1971) Multiple Sampling in Non-Response Problems. Journal of the American Statistical Association, 66, 583-586. http://dx.doi.org/10.1080/01621459.1971.10482310

[7] Khare, B.B. (1987) Allocation in Stratified Sampling in Presence of Non-Response. Metron, 45, $213-221$.

[8] Khan, M.G.M., Khan, E.A. and Ahsan, M.J. (2008) Optimum Allocation in Multivariate Stratified Sampling in Presence of Non-Response. Journal of the Indian Society of Agricultural Statistics, 62, 42-48.

[9] Varshney, R., Ahsan, M.J. and Khan, M.G.M. (2011) An Optimum Multivariate Stratified Sampling Design with NonResponse: A Lexicographic Goal Programming Approach. Journal of Mathematical Modeling and Algorithms, 65, 291-296.

[10] Fatima, U. and Ahsan, M.J. (2011) Nonresponse in Stratified Sampling: A Mathematical Programming Approach. The South Pacific Journal of Natural and Applied Sciences, 29, 40-42.

[11] Varshney, R., Najmussehar and Ahsan, M.J. (2012) An Optimum Multivariate Stratified Double Sampling Design in Presence of Non-Response. Optimization Letters, 6, 993-1008.

[12] Raghav, Y.S., Ali, I. and Bari, A. (2014) Multi-Objective Nonlinear Programming Problem Approach in Multivariate Stratified Sample Surveys in Case of Non-Response. Journal of Statistical Computation and Simulation, 84, 22-36. http://dx.doi.org/10.1080/00949655.2012.692370

[13] Duffin, R.J., Peterson, E.L. and Zener, C. (1967) Geometric Programming: Theory \& Applications. John Wiley \& Sons, New York.

[14] Zener, C. (1971) Engineering Design by Geometric Programming. John Wiley \& Sons, New York.

[15] Beightler, C.S. and Philips, D.T. (1976) Applied Geometric Programming. Wiley, New York.

[16] Davis, M. and Schwartz, R.S. (1987) Geometric Programming for Optimal Allocation of Integrated Samples in Quality Control. Communications in Statistics_-Theory and Methods, 16, 3235-3254. http://dx.doi.org/10.1080/03610928708829568

[17] Ahmed, J. and Bonham, C.D. (1987) Application of Geometric Programming to Optimum Allocation Problems in Multivariate Double Sampling. Applied Mathematics and Computation, 21, 157-169. http://dx.doi.org/10.1016/0096-3003(87)90024-5

[18] Ojha, A.K. and Das, A.K. (2010) Multi-Objective Geometric Programming Problem Being Cost Coefficients as Con- 
tinuous Function with Weighted Mean. Journal of Computing, 2, 2151-9617.

[19] Maqbool, S., Mir, A.H. and Mir, S.A. (2011) Geometric Programming Approach to Optimum Allocation in Multivariate Two-Stage Sampling Design. Electronic Journal of Applied Statistical Analysis, 4, 71-82.

[20] Shafiullah, Ali, I. and Bari, A. (2013) Geometric Programming Approach in Three-Stage Sampling Design. International Journal of Scientific \& Engineering Research (France), 4, 2229-5518.

[21] Zadeh, L.A. (1965) Fuzzy Sets. Information and Control, 8, 338-353. http://dx.doi.org/10.1016/S0019-9958(65)90241-X

[22] Bellman, R.E. and Zadeh, L.A. (1970) Decision-Making in a Fuzzy Environment. Management Sciences, 17, B141B164. http://dx.doi.org/10.1287/mnsc.17.4.B141

[23] Tanaka, H., Okuda, T. and Asai, K. (1974) On Fuzzy-Mathematical Programming. Journal of Cybernetics, 3, 37-46. http://dx.doi.org/10.1080/01969727308545912

[24] Zimmermann, H.J. (1978) Fuzzy Programming and Linear Programming with Several Objective Functions. Fuzzy Sets and Systems, 1, 45-55. http://dx.doi.org/10.1016/0165-0114(78)90031-3

[25] Biswal, M.P. (1992) Fuzzy Programming Technique to Solve Multi-Objective Geometric Programming Problems. Fuzzy Sets and Systems, 51, 67-71. http://dx.doi.org/10.1016/0165-0114(92)90076-G

[26] Verma, R.K. (1990) Fuzzy Geometric Programming with Several Objective Functions. Fuzzy Sets and Systems, 35, 115-120. http://dx.doi.org/10.1016/0165-0114(90)90024-Z

[27] Islam, S. and Roy, T.K. (2005) Modified Geometric Programming Problem and Its Applications. Journal of Applied Mathematics and Computing, 17, 121-144.

[28] Islam, S. (2010) Multi-Objective Geometric Programming Problem and Its Applications. Yugoslav Journal of Operations Research, 20, 213-227. http://dx.doi.org/10.2298/YJOR1002213I

[29] Tiwari, R.N., Dharman, S. and Rao, J.R. (1987) Fuzzy Goal Programming-An Additive Model. Fuzzy Sets and Systems, 24, 27-34. http://dx.doi.org/10.1016/0165-0114(87)90111-4

[30] Lindo Systems Inc. (2013) LINGO User’s Guide. Lindo Systems Inc., Chicago. 